\title{
Topology Optimization - a Variational Formulation of the Problem and Example Application
}

\author{
Ryszard Kutyłowski ${ }^{1 *}$, Marek Szwechłowicz ${ }^{1}$ \\ ${ }^{1}$ Faculty of Civil Engineering, Wrocław University of Science and Technology, Wybrzeże St. Wyspiańskiego 27, 50-370 Wrocław, \\ Poland \\ * Corresponding author, e-mail: ryszard.kutylowski@pwr.edu.pl
}

Received: 09 March 2019, Accepted: 05 August 2019, Published online: 13 January 2020

\begin{abstract}
A variational formulation of the topology optimization problem is presented. A strain energy functional, being an equivalent of compliance, was minimized while constraints were imposed on the body mass. A global mass constraint and a local constraint on the amount of mass accumulated in a single material point of the body were adopted. A penalization procedure was defined and implemented in the optimization process to speed up the latter. The procedure in the successive optimization process steps translocates mass within the design domain, from the less strained areas to the more strained ones. The optimization process was described as a series of sequences of topologies determined using various control parameters, including different threshold functions. This means that the optimization process is characterized by a sequence of objective functional values approaching a minimal value. Various functions updating Young's modulus were considered. Primarily the updating method referred to as SIMP was adopted. Three ways of using the discrete strain energy value to update Young's modulus in the considered material point were taken into account. These were: the amount of energy accumulated in the preceding step, the sum of the amounts of energy from all the preceding steps and the average amount of energy from the last two steps. In order to ensure the global limiting condition a mass constancy satisfaction procedure was incorporated into the algorithm. The algorithm procedures are described in detail. Finally, the algorithm was used to analyze selected problem relating to the pavement structure and the structure of tall buildings.
\end{abstract}

\section{Keywords}

topology optimization, minimum compliance, mass constraint, pavement structure analysis, tall buildings

\section{Introduction}

Topology optimization is a process aimed at obtaining an optimum topology of a structure. The following terms and quantities are used in this paper:

- an optimal solution is a solution characterized by a minimum value of the objective functional which in the considered case is the strain energy determined as a result of an optimization process;

- a design domain is an area within which the optimization process is conducted,

- available mass is the amount of mass to be distributed within the design domain. The amount of available mass is determined separately for each process, which means that an optimal topology which takes into account the amount of available mass is determined in each process separately;

- a material point is a point belonging to a design domain with explicitly specified coordinates and explicitly specified material parameters. In addition, it is assumed that a material point can have infinitesimal volume $d V$. For the numerical implementation of the problem it is assumed that there is a mutual correspondence between a material point as a physical quantity and its numerical model, i.e. a finite element;

- a topology is the shape of a structure, i.e. a discrete distribution of material in the design domain.

The optimization process is conducted within an initially defined design domain $\Omega$ which remains invariable in the course of the process. Displacement and stress boundary conditions are defined on the design domain edge. The process of optimization consists in translocating material within the design domain until an optimal, according to the adopted objective function, distribution of the material is obtained. The result of the optimization process is an optimal distribution of material, as symbolically shown 


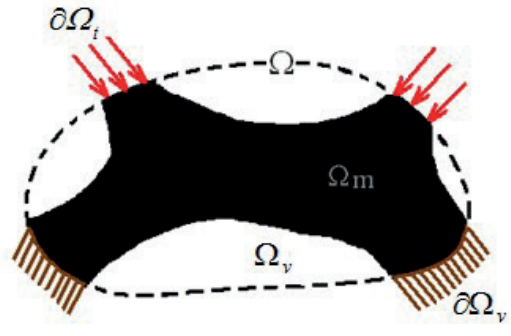

Fig. 1 Design domain $\Omega$

in Fig. 1, where in domain $\Omega$ one can distinguish subdomain $\Omega_{m}$ filled with material and subdomain $\Omega_{v}$ in which there is no material. The paper is generally based on the artificial density approach [1-4] and also on the author's previous work [5].

\section{Variational approach to the topology optimization problem}

In this study the topology optimization problem is presented in a variational formulation. A variational integral functional describing the compliance of a structure is used for this purpose. Then the character of the optimization process, consisting in searching the particular sequences of solutions for an objective functional with a minimal value, is presented. The functional describes a construction which under the imposed constraints is maximally stiff from the physical point of view.

The Lagrange theorem on minimum potential energy is applied. The potential energy of the external load and of the body forces, equivalent to the compliance of the construction, is expressed by the relation:

$$
\Pi^{E}=\int_{\Omega} \mathbf{X} \mathbf{v} d \Omega+\int_{\partial \Omega_{t}} \mathbf{t v} d s
$$

where $\mathbf{X}$ is the tensor of the body forces, $\mathbf{t}$ is the tensor of the surface forces and $\mathbf{v}$ is the displacement tensor. In the literature on the subject, and also in this paper, the topology problem is typically considered taking into account the surface forces, but neglecting the body forces. Under the action of the surface forces, located inside or on the edge of the assumed design domain, the potential energy of the internal forces accumulates in the design domain (strain energy). This energy can be described by the relation:

$$
\Pi^{I}=\frac{1}{2} \int \mathbf{e}^{\mathrm{T}} \mathbf{C e} d \Omega,
$$

where $\mathbf{C}$ is an elasticity tensor and $\mathbf{e}$ is a strain tensor. In the above relations, tensor $\mathbf{X}$ and tensor $\mathbf{C}$ can be presented in the form of an explicit function of respectively construction material density and an elasticity constant called Young's modulus. A solution of the optimization problem is usually sought assuming that the Young modulus in a given material point in a given (the $j$-th) optimization step is a function of the material density in the $j$-1th step:

$E_{j}=E\left(\rho_{j-1}(x)\right)$,

where $E$ is the Young modulus of the material from which the structure is to be built. Thanks to this relation, mass can be translocated within the design domain in the course of optimization. Considering the equivalence between compliance and strain energy:

$\Pi^{E}=2 \Pi^{I}$,

construction strain energy can be assumed to be equivalent to compliance. Therefore strain energy can be adopted as the objective functional $(F)$. Similarly as compliance, this energy should be minimized, which means that such a state of the design domain will be sought for which strain energy will be minimum under the prescribed load and boundary conditions. Considering Eq. (3), the objective functional depends on the density in each of the material points:

$F(\rho(x))=\int_{\Omega} \mathbf{e}^{\mathrm{T}} \mathbf{C}(\rho(x)) \mathbf{e d} \Omega$.

One should bear in mind that constraints were imposed on the mass of the body situated within the design domain. This means that each time the problem is solved under the assumption that a strictly defined mass, referred to as available mass $m_{0}$ equal to

$m_{0}=\alpha m, \quad 0<\alpha<1$,

where

$m=V \rho$

is available for the given optimization process.

In the above formulas, $V$ is the volume of the design domain and $\rho$ is the density of the material from which the structure is made. Coefficient $\alpha$ is a mass reduction coefficient. It specifies what part of mass $m$ completely filling volume $V$ has been allocated for building the structure in the given process of designing the latter, called an optimization process. The above constraint is a global constraint applying to the structure and the design domain as a whole and it is formulated as the starting constraint imposed on the optimization process. In the successive steps of the optimization process this global constraint can be defined as follows:

$m_{j}=m_{0}$, 
which means that this initial constraint must be satisfied in each $j$-th step of the optimization process. Therefore when formulating the optimization problem with the imposed constraint, the objective functional with constraints imposed on mass has this form:

$$
F(\rho(x), \lambda)=\int_{\Omega} \mathbf{e}^{\mathrm{T}} \mathbf{C}(\rho(x)) \mathrm{ed} \Omega+\lambda\left(\int_{\Omega} \rho(x) \mathrm{d} \Omega-m_{0}\right) .
$$

Function $\rho(x)$ has values denoted as $\rho_{x}$. These are body densities in any material point of domain $\Omega$ defined by coordinate $x$ while $\lambda$ is a Lagrange multiplier. In the above equation there are two design variables: $\rho(x)$ and $\lambda$. The solution of the problem consists in minimizing the objective function defined as follows:

$$
\begin{aligned}
& \min F(\rho(x), \lambda)= \\
& \min \left\{\begin{array}{l}
\int_{\Omega} \mathbf{e}^{\mathrm{T}} \mathbf{C}(\rho(x)) \mathbf{e d} \Omega+ \\
\lambda\left(\int_{\Omega} \rho(x) \mathrm{d} \Omega-m_{0}\right)
\end{array}\right\} .
\end{aligned}
$$

As a result of optimization, in each next step one obtains a distribution of design variable $\rho(x)$ over the whole design domain. Since generally this variable may assume different values, in order for the optimization process to be effective additional, this time local constraints were imposed on density values:

$$
0 \leq \rho_{x} \leq \rho,
$$

which means that the considerations apply to a real material from which the construction is to be built and in each step a local constraint is imposed separately on each material point of the body. To sum up, an objective functional for the topology optimization problem has been formulated. The optimization process is carried out for a boundary value problem for which the system of equations

$$
\begin{aligned}
& \operatorname{div} \boldsymbol{\sigma}=0 \\
& \boldsymbol{\sigma}=\mathbf{C e} \\
& \mathbf{e}=\frac{1}{2}\left(\nabla \mathbf{v}+\nabla \mathbf{v}^{T}\right) \\
& \mathbf{v}=0 \text { on } \partial \Omega_{v} \\
& \boldsymbol{\sigma} \mathbf{n}=\mathbf{f} \text { on } \partial \Omega_{r}
\end{aligned}
$$

has a unique solution in the field of displacements v. A solution of the topology optimization problem is obtained by minimizing the objective functional with regard to design variables $\rho$ and $\lambda$ : $\frac{\partial F}{\partial \rho}=0 \Rightarrow \frac{\partial\left(\mathbf{e}^{\mathrm{T}} \mathbf{C}(\rho(x)) \mathbf{e}\right)}{\partial \rho}+\lambda=0$,

$\frac{\partial F}{\partial \lambda}=0 \Rightarrow \int_{\Omega} \rho(x) \mathrm{d} \Omega=m_{0}$.

The problem was solved assuming the design domain to be invariable and the load to be independent of design variable $\rho$. Hence the first of Eq. (13) assumes the form:

$\mathbf{e}^{\mathrm{T}} \frac{\partial \mathbf{C}(\rho(x))}{\partial \rho} \mathbf{e}+\lambda=0$.

Taking into account the fact that according to Eq. (3) the Young modulus in the considered material point in the current step depends on the material density in this point in the preceding step and performing the above integration and in addition, multiplying and dividing the derivative obtained in this way by $\rho$, as well as multiplying both sides by $\rho$ one gets the following linear dependence between the strain energy, expressed here in a discrete form (for the considered material point), and the density in this point:

$\mathbf{e}^{\mathrm{T}} \mathbf{C}(\rho(x)) \mathbf{e}=\rho \frac{\partial\left(\mathbf{e}^{\mathrm{T}} \mathbf{C}(\rho(x)) \mathbf{e}\right)}{\partial \rho}$.

The factor of proportionality in this relation is a derivative of strain energy over density. It emerges from the above equation that the optimization process will lead to a reduction in the density of the construction material in these parts of the design domain in which strain energy will diminish (due to the lower strain of these parts of the design domain in comparison with its other areas) and vice versa the optimization process will result in increased construction material density in the parts of the design domain in which strain energy will increase, where, because of the increasing strain of the material, it will be necessary to incorporate a relatively larger amount of material, which will result in increased material density. The differentiation of density is usually effected using the well known following relation updating Young's modulus in the next $j$-th optimization step:

$E_{j}=E\left(\frac{\rho_{j-1}}{\rho}\right)^{p}$.

In order to increase the effectiveness of the optimization process based on Eq. (16) a penalization procedure was applied. Threshold functions are the main part of the penalization procedure which in a given step defines the negligibly small degree of material strain. The penalization procedure firstly eliminates material from the 
relatively less strained areas (from which material has not been removed by the procedure based on Eq. (16)) and secondly, ensures the translocation of this removed material within the design domain.

The use of threshold functions can be interpreted as the exploitation of the Powell method with a shifted penalization function because the threshold functions are functionally linked with the number of the optimization process step Eq. (19). Consequently, the threshold function value shifts (in this case, increases) in the course of the optimization process, whereby the mass translocates increasingly more gradually than when constant threshold values are used. Constant threshold function values have been widely used in the literature on topology optimization (for example in [6]), but their effectiveness is lower than that of threshold functions.

What is important is that the adopted penalization procedure results in the satisfaction of the global limiting condition (in this case, imposed on the body mass, but at this stage of analysis expressed by strain energy). This is owing to the translocation of mass within the design domain. Moreover, in the course of the optimization process local constraining condition (Eq. (11)) must be satisfied in each of the material points. Thus in the considered problem a global-local constraining condition occurs. Since the nominal sum of strain energy (i.e. of mass) in the successive optimization steps is almost always different from the value of the available mass, all the mass density values in the particular points need to be appropriately rescaled so that the total mass from all the points is equal to the available mass. This scaling is part of the mass constancy satisfaction procedure (PSSM). It ensures the satisfaction of the global constraining condition expressed by Eq. (8), according to which the total mass within the whole design domain must be equal to the available mass. It also ensures a material distribution which meets the following criterion: in none of the points material density exceeds the density of the material used to build the construction.

According to Eq. (15), density considerations are based on the distribution of strain energy in the structure. Lower local condition (Eq. (11)) is always satisfied since the energy accumulated in any area of the structure is always non-negative. Normalized quantities are used in the optimization process and such quantities will be used here. Thus Eq. (11) can already be considered to be an inequality normalized relative to $\rho$. It appears from computations that normalized strain energy values can considerably differ between some areas. However, even if in the given optimization step some areas differ in the absolute value of the strain energy accumulated in them, inequality (Eq. (11)) concerning density will be satisfied. By using normalized values one avoids a situation when the upper bound of material density value $\rho$ could be exceeded. The values of some densities $\rho_{x}$ in Eq. (11) can be close to the upper bound while some of them can be close to zero. This may cause difficulties in solving the boundary problem due to the weak conditioning of the problem, which is based on Eq. (16) for the updating of Young's modulus in each successive step of the optimization process. Therefore in order for the problem to be well conditioned the following additional constraints modifying condition (Eq. (11)) are assumed:

$0 \leq \rho_{d} \leq \rho_{x} \leq \rho$,

where $\rho_{d}$ is the lower bound imposed on density. Since according to Eq. (15) density is proportional to the strain energy accumulated in the body, let us return to considering the strain energy values in the particular areas of the construction. The values define the degree of strain of the construction material in a given area. If the strain energy value in some area is relatively very small, this physically means that the area does not undergo deformation and so it is not strained. Therefore the question arises whether in this area material is needed at all. If the answer is negative, this means that material should be removed from this area in the next stage of the optimization process. This can be done by equating this area's density to zero. If the value equated to zero were then used in Eq. (16), this would result in the bad conditioning of the problem. It is apparent that by adopting $\rho_{d}$ in this area one can preserve the good conditioning of the problem. Thus condition (Eq. (17)) ultimately becomes

$\rho_{d} \leq \rho_{x} \leq \rho$,

where $\rho_{x}$ is the value of function $\rho(x)$ in a range of $\left[\rho_{d}, 1\right]$. Then the degree of material strain, which may be considered as negligibly small in the next steps, needs to be defined. This is done using the threshold function and applying the penalization procedure to the whole process of mass translocation, as briefly described above. The threshold values in the particular process steps are variable and increase in the successive steps, ensuring the removal of material with ever higher relative densities, whereby problem convergence can be achieved relatively quickly. The threshold function $(T F)$ can be generally written as: 
$F P=F P(n r)$,

where $n r$ stands for the number of the optimization process step. Let us consider now a way of exploiting the threshold functions in the discrete process of topology optimization. In each successive $j$-th step of a given optimization process the value of threshold function $F P_{j}$ is determined. The objective functional defined by Eq. (10) can be considered discretely. For the next $j$-th step of the process it is denoted as $F_{j}$ and it is an element in a sequence of functionals in the process of optimization. The sequence is a set of values tending to satisfy Eq. (10), i.e. a minimum value of the objective functional at fixed values of the control parameters is obtained. In this case, the control parameter is the threshold function. The value of the objective functional, i.e. the value of strain energy, is understood in discrete terms as the sum of the values of the strain energy accumulated in the particular subareas of the design domain. The subareas, understood discretely, can be, e.g., individual finite elements in a numerical analysis. Thus the value of objective functional $F_{j}$ in the $j$-th step is the following sum:

$F_{j}=\sum_{i} F_{j}^{i}$,

where $F_{j}^{i}$ is the value of the objective functional (strain energy) in the $i$-th subarea of the design domain. Let us define function $H$ :

$H\left(F_{j}^{i}-F P_{j}\right)=\left\{\begin{array}{l}1 \text { dla } F_{j}^{i}-F P_{j}>0 \\ 0 \text { dla } F_{j}^{i}-F P_{j} \leq 0\end{array}\right.$.

Then the value of functional $F_{j}$ can be expressed as:

$F_{j}=\sum_{i} H \cdot F_{j}^{i}+R F P_{j}$,

where

$$
R F P_{j}=\sum_{i} F_{j}^{i}-\sum_{i} H \cdot F_{j}^{i},
$$

in the given $j$-th step is the sum of the strain energy values considered to be negligibly small in the particular subareas. Since according to Eq. (15), the density of the material accumulated in a given subarea is proportional to the strain energy accumulated in it, the density of the construction material will be taken into account in further considerations. Hence $R F P_{j}$ is physically interpreted as the total mass removed from the construction and its removal is justified by the fact that this material is useless for the construction's stiffness in the $j$-th step. When the unneeded material has been removed, the problem of satisfying global constraining Eq. (8) arises. However, as mentioned above, since for the time being the analysis is conducted for strain energy values, the satisfaction of Eq. (8) means that after the operation of the threshold function the total strain energy must be equal to that before the threshold function operation. Therefore $m e_{j}$, representing the mass being the equivalent of the strain energy accumulated in the construction, will be used instead of $m_{j}$ for the needs of the operation of the threshold function in further considerations. It is assumed that the quantities expressed by Eqs. (20)-(23) can be regarded as normalized quantities. Then $R F P_{j}$ can be interpreted as a part of mass $m e_{j}$, which can be called $m e_{j}^{R}$. Since it is necessary to satisfy Eq. (8), material with mass $m e_{j}^{R}$ should be distributed within the design domain. The whole mass $m e_{j}^{R}$ must be subject to distribution. The material can be distributed uniformly among all the subareas or it can be selectively distributed only among some subareas satisfying certain criteria. Selective distribution algorithms are often convergent faster than the ones which distribute material among all the subareas. Depending on the applied distribution criterion, one can obtain different solution convergence and different solutions (topologies). This is so since also the distribution method is a control parameter which has a bearing on the solution. Fast convergent solutions are not always optimal since they are not always characterized by a minimum strain energy value.

The following principal methods can be distinguished:

a) distribution among all the subareas with density higher than $\rho_{d}$,

b) distribution among all the subareas with density higher than the functionally determined limit density value.

In case b), this can be a constant value or a value dependent on the number of the optimization process step, which seems to be logical since as the step number increases, the distribution increasingly resembles a material/void distribution and an increasingly larger number of subareas has the density of the construction material, but at the same time an ever larger number of subareas is not filled with material. In this situation convergence can be speeded up using a distribution whose functional description is connected with the optimization process step number.

Considering Eq. (8) one can write:

$m e_{j}=m e_{j}^{P}+m e_{j}^{R}$,

where $m e_{j}^{R}$ is, as described above, the mass (in terms of the strain energy value) removed from the little strained subareas and $m e_{j}^{P}$ is the remaining mass (in terms of the 
strain energy value) distributed in the design domain in the $j$-th step. Then when the mass constancy condition is satisfied, Eq. (24) becomes:

$m_{0}=m_{j}=m_{j}^{p}+m_{j}^{R}$,

where $m e_{j}^{R}$ is, as described above, the (rescaled for the available mass) mass removed from the little strained subareas and $m e_{j}^{P}$ is the remaining mass (also rescaled) distributed in the design domain in the $j$-th step.

Hence the modified objective functional has this form:

$\min F(\rho(x), \lambda)=$

$\min \left\{\int_{\Omega} \mathbf{e}^{\mathrm{T}} \mathbf{C}(\rho(x)) \mathbf{e d} \Omega+\lambda\left(\int_{\Omega} \rho(x) \mathrm{d} \Omega-\left(m_{j}^{p}+m_{j}^{R}\right)\right)\right\}$.

Consequently, the second relation in Eq. (13) becomes:

$\frac{\partial F}{\partial \lambda}=0 \Rightarrow \int_{\Omega} \rho(x) \mathrm{d} \Omega=m_{j}^{p}+m_{j}^{R}$.

In this way several topology sequences determined using different control parameters (including different threshold functions) can be obtained. Let us define set $Z$ whose elements are subsets $T_{k}$, where $k$ stands for the topology optimization process number:

$Z=\left\{T_{1}, T_{2}, T_{3}, \ldots, T_{M}\right\}$,

where $M$ is a finite number of obtainable sets of topologies. Let us assume that the topology optimization process is controlled by a threshold function. The topology subsets isolated from set $Z$ are defined as:

$Z^{F P}=\left\{T_{1}^{F P}, T_{2}^{F P}, T_{3}^{F P}, \ldots, T_{N}^{F P}\right\}$,

where $N$ is a finite number of the considered threshold functions ( $m=1,2, \ldots, N)$. Each of the $T_{m}^{F P}$ sets consists of a sequence of the topologies obtained in the given process:

$T_{m}^{F P}=\left\{T_{m}^{F P, 1}, T_{m}^{F P, 2}, T_{m}^{F P, 3}, \ldots, T_{m}^{F P, S}\right\}$,

where $S$ is the number of the step in which the optimal topology was obtained (step number $j=1,2, \ldots, S$ ). To each element of sequence $T_{m}^{F P, j}$, i.e. to each of the topologies, an objective functional value is assigned. Therefore, similarly as (3.30), one can write:

$F_{m}^{F P}=\left\{F_{m}^{F P, 1}, F_{m}^{F P, 2}, F_{m}^{F P, 3}, \ldots, F_{m}^{F P, S}\right\}$.

In the case of relation (Eq. (30)), there is an ordered sequence of topologies changing so that in step $S$ the material distribution in the design domain becomes of the material/void type or is very similar to the latter. Relation
(Eq. (31)) describes an ordered sequence of objective functional values approaching the minimum value in step $S$. As a result of the changes made to Eq. (26) relative to Eq. (10) the character of the objective functional, including that of its continuity, did not change. Hence, in the general case, depending on the control parameters one obtains solutions which satisfy:

$F(\rho(x), \lambda) \Rightarrow \inf F(\rho(x), \lambda)$.

In a special case, when one of the control parameters (e.g. the threshold function) is analyzed, there also exists a sequence of objective functionals $F^{F P}$ to which topologies $Z^{F P}$ correspond, and this sequence $\left(T_{m}^{F P}\right)$ is convergent to the lower bound in accordance with Eq. (32). Each topology is described by the distribution of density function $\rho(x)$. The sequence of density functions in the successive steps is denoted as $\rho_{j}(x)$. It is a set of the densities of the particular $i$-th subareas in the considered step $j$ :

$\rho_{j}(x)=\left\{\rho_{j}^{1}, \rho_{j}^{2}, \rho_{j}^{3}, \ldots, \rho_{j}^{n}\right\}$,

where $n$ is the number of subareas in area $\Omega$.

In topology optimization one usually applies the principle that the updating of Young's modulus in the $j$-th step proceeds according to Eq. (16) on the basis of the material density in the considered subarea in the previous step, i.e. $j-1$.

Several ways of updating are considered in this paper. One of them is the way represented by Eq. (16). The following updating method, taking into account the density distributions from the preceding steps, was also used:

$E_{j}=E\left(\frac{\left|\sum_{s} \rho_{s}\right|}{\rho}\right)^{p}$,

where $s$ ranges from 1 to step $j-1$. The method is based on [5].

According to what was mentioned above Eq. (17), quantity $\rho$ in the denominator should be treated as a normalized quantity. Relation (Eq. (34)) applies to each subarea of domain $\Omega$ individually, which means that the Young modulus is discretely updated:

$E_{j}^{i}=E\left(\frac{\left|\sum_{s} \rho_{s}^{i}\right|}{\rho}\right)^{p}$,

where $i$ ranges from 1 to a finite number of subareas $n$. As a result, the updating process is stable and convergent. 
One more way of updating Young's modulus was considered. According to it, a density value calculated for the averaged energy from the two preceding steps is used in a given step. This is described in detail in Section 3.2.

Many other approaches in topology optimization are considered. Parallel to presented minimum compliance approach among others there are metaheuristic algorithms. As an example the Ant Colony Optimization approach can be mentioned [7], where similar to presented approach, the stiffest structure with a certain amount of material, based on the element's strain energy was obtained.

\section{Finite element method algorithm in topology optimization}

\subsection{Introduction}

Thanks to the use of the finite element method (FEM) in computer-aided engineering analyses relatively accurate results, which would be difficult to obtain analytically, can be quickly obtained. By discretizing a continuous area into a finite number of subareas (finite elements) and using appropriate computational algorithms one obtains approximate solutions characterized by good agreement between the results of computer simulations, analytical solutions and experiments.

Today commercial computer programs based on FEM incorporate modules for optimizing the geometry of structural elements being designed. As the variables in the programs, parameters describing geometry (dimensions, surface area) are adopted and constraints are imposed on the magnitude of internal forces (stresses) or displacements. The weight of the object being designed is usually adopted as the objective function.

In this research an in-house algorithm and program (based on FEM and running in Matlab) were used for computations. Considered optimization problem was solved using the Optimality Criteria method.

Very seldom descriptions of the algorithms and codes used in such computational programs can be found in the literature on topology optimization. Additionally many researches work on development of efficient computational procedures for topology optimization. The main and most cited work in which such an algorithm is described is [8]. One can also find there the program code whereby the computational algorithm can be traced step by step, which indirectly makes it possible to compare the end results published there with one's own results. This work has been an inspiration for others. As examples, two papers referring to it are briefly discussed below.
In [9] it is presented a new approach to the computing algorithm for topology optimization. This paper is indicating the differences in the computing algorithms used in the "99 lines" program and the "88 lines" program. The changes contributing to a reduction in operating memory use for computations and to a reduction in computing time were described in detail. In 2011, the entire code of the topology optimization program was published in [9]. The computing algorithm presented there was written in Matlab and used to perform analyses of a cantilever and a freely supported beam, included in the paper. The topologies obtained for different static diagrams at different mass reduction coefficients (different volumetric mass shares) were compared with the ones yielded by the program based on the code published by Sigmund in [8] showing some similarities.

It should be added that in one of the chapters [4] monograph the operation of the topology program was described by writing out the codes. Besides the 99 lines code presented earlier also the computing algorithms based on this code were described in this book. The 105 lines program, which by expanding the 99 lines code one can use to perform computations for mechanisms and the 91 lines code (also based on the 99 lines code) which makes it possible to carry out analyses of heat conduction, elastic torsion, magnetic conductance and other problems, were presented there.

Computing algorithms for topology optimization are also written in Mathematica [11] to obtain the optimal solutions for Michell's trusses. This approach is based on linear programming.

\subsection{Finite element method algorithm}

A finite element method algorithm, based on the considerations presented in Section 2, is described below. It should be noted that mutual correspondence between a finite element and a material point of a body, represented by an infinitesimal area of the body, is assumed. This assumption is needed since variable material parameters will used, which in FEM is expressed by the changing parameters of the particular finite elements.

In accordance with Eq. (4), strain energy is adopted as the equivalent of compliance. In FEM notation, the total strain energy $\left(\Pi_{j}^{I}\right)$ in each $j$-th step is the sum of the energies determined for the particular $i$-th elements $\left(\Pi_{j}^{I}\right)$ and it can be described by the relation:

$$
\Pi_{j}^{I}=\sum_{i=1}^{n} \Pi_{j}^{i}=\sum_{i=1}^{n}\left(\delta_{j}^{i}\right)^{T} \cdot k_{j}^{i} \cdot{ }^{\prime}{ }_{j}^{i}
$$


where $\delta_{j}^{i}$ is a nodal parameters matrix, $k_{j}^{i}$ - a matrix of the stiffness of the $i$-th element in the $j$-th step, and $n$ is the number of finite elements into which the design domain has been divided. The normalized strain energy value in the $i$-th element is expressed as:

$\overline{\Pi_{j}^{i}}=\frac{\Pi_{j}^{i}}{\Pi_{j \max }^{i}}$,

where $\prod_{j}^{i}$ is the amount of strain energy accumulated in the $i$-th element. According to Eq. (15), the material density value in the $i$-th element in the $j$-th step is proportional (with an accuracy of the constant) to the normalized strain energy in this element in this step:

$\overline{\rho_{j}^{i}}=\overline{\Pi_{j}^{i}} \rho$,

where, let us remind ourselves, $\rho$ is the density of the material from which the construction is to be built. In order to make comparative analyses possible the density value was normalized:

$\rho_{j}^{i}=\frac{\overline{\rho_{j}^{i}}}{\overline{\rho_{j \max }^{i}}}$,

which means that $\rho_{j}^{i}$ is the normalized density in the $i$-th element in the $j$-th step of the optimization process. In each step $j, \rho_{j \max }^{i}$ is the maximum density value in the whole design domain and it corresponds to density $\rho$ of the construction material. The normalized relative density expressed by Eq. (39) will be used in further considerations. For the sake of simplicity, it will be referred to as simply density. In the general case, the values of such density will always be in the range of $[0,1]$. In practice, some values of $\rho_{j}^{i}$ in a given step may be very low (so low that the problem will no longer be well-conditioned in the numerical sense), but higher than zero. Consequently, it will be necessary to use Eq. (17), and then Eq. (18), i.e. to introduce $\rho_{d}$, (the lower bound of density) whose value is matched to ensure good problem conditioning.

In the course of FEM topology optimization a discrete distribution of material of varied density will be obtained in the adopted design domain. In the literature such a material with density in the range of $(0,1]$ is referred to "fictional material".

Towards the end of a given optimization process in some subareas of the design domain there will be material which density will be very high, while in other subareas there will be a material which density will be very low (insignificant from the structural point of view), which is needed to ensure good problem conditioning. Sometimes even when the optimization process ends, there may be material with density slightly lower than that of the material intended for the construction, i.e. with densities below unity, but close to unity.

A discrete distribution of mass within the design domain will be referred to as a topology. Topologies, i.e. distributions of density values in the particular components may be presented in the numerical notation (in the range of $[0,1]$ ) or in the shades-of-grey notation (from white, through shades of grey to black). The white color will be assigned to 0 while black will be assigned to 1 . The shades of grey will be appropriately scaled and they will correspond to numerical values from the interval of $0-1$ (Fig. 2). Thus in the drawings (topologies) presented here the black color will represent density equal to one (Fig. 3a), i.e. the element will be filled with the material from which the construction is to be built. The white color (Fig. 3a) will represent density amounting to zero, i.e. the absence of material. The grid visible in some of the drawings is the finite element mesh. Solutions will also be presented in

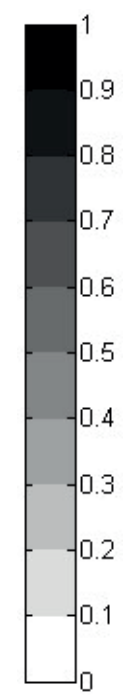

Fig. 2 Adopted grey scale

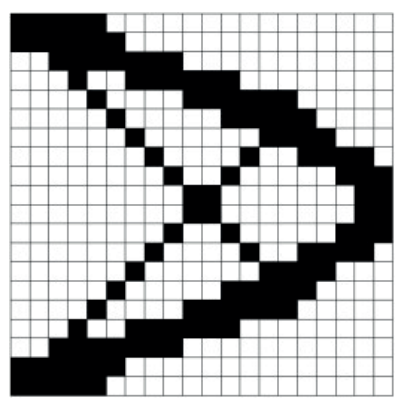

(a)

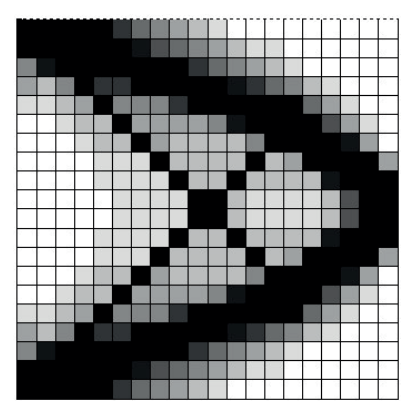

(b)
Fig. 3 0/1 topology (a); shades-grey topology (b) 
shades of grey (Fig. 3b). In such cases, "rarefied" density will occur, i.e. besides the number one (black) and zero (white) there will be values from the interval of $0-1$.

To sum up, the optimum solution, i.e. one satisfying the minimal strain energy condition, will usually be characterized by a black and white distribution with some grey. This means that besides material and voids some of the elements will include material whose density will usually be very close to that of the construction material. Sometimes one manages to obtain a solution satisfying both the minimal strain energy condition and the material/void distribution condition. Since the designer is usually required to submit topologies of the material/void type, the latter case meets this criterion whereas in the former case one should do postprocessing which will usually make up the missing material in the elements whose density is lower than that of the construction material.
Let us present now the computing algorithm. A schematic block diagram of the algorithm is shown in Fig. 4. The algorithm includes Eqs. (36)-(39). Since the formulas are used in the iterative process, in some places in the diagram the symbols and indices have been slightly modified for the sake of graphical representation. A fournode tetragonal finite element is used in the program. The displacement state of the element is described with eight nodal parameters: two in each node (displacements in mutually orthogonal directions).

Fig. 4a shows the algorithm's preliminary stage, i.e. the starting point for the topology optimization process. At this stage the boundary problem is solved and a displacement matrix is obtained. The latter then allows one to discretely determine the strain energy in each finite element separately, whereby a discrete distribution of the construction strain is obtained. Considering Eq. (38), material density

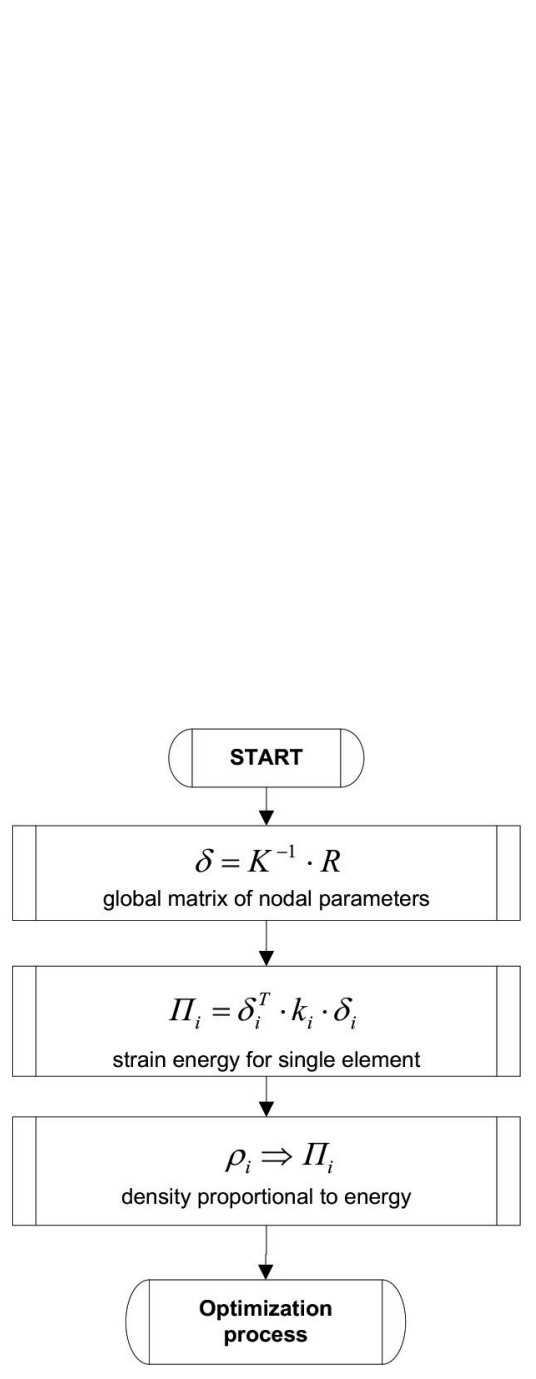

(a)

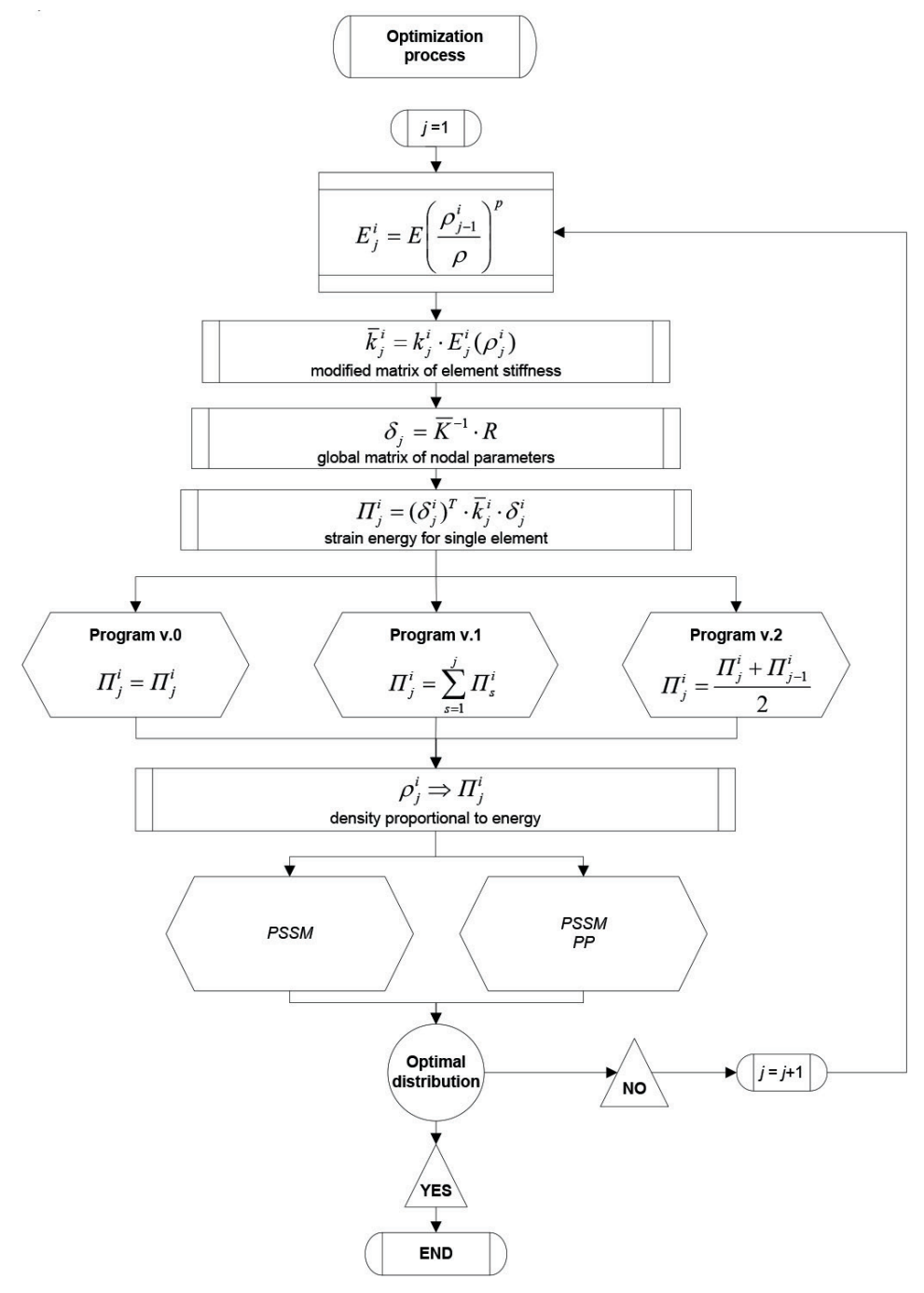

(b)

Fig. 4 Schematic block diagram of preliminary stage (a); optimization process (b) 
in each element is proportional to the strain energy accumulated in it. Consequently, one gets the distribution of density in the adopted design domain. This density will be used in the first step of the topology optimization process to update Young's modulus. One should remember that after the strain energy value is determined it is normalized and subsequently exclusively normalized values are used.

Then the topology optimization starts (Fig. 4b). It is iteratively run in successive steps denoted with the letter $j$. In each of the steps the updated value of Young's modulus is discretely determined on the basis of the density distribution in the previous step, as shown for Eq. (16) in Fig. 4b. Using the updated value of Young's modulus characterizing the elasticity of the given element, one determines stiffness matrices for the particular elements in the given step. The matrices are used to determine modified global stiffness matrix $\bar{K}$. Then solving system of equations $\bar{K} \cdot \delta=R$ one determines the global matrix of nodal parameters $(\delta)$. which in each successive $j$-th step will be denoted as $\delta_{j}$. Coming down to the level of the individual elements and using the values of the nodal parameters characterizing the displacement state of the given element one calculates the value of the strain energy accumulated in each individual element.

The algorithm for determining the normalized strain energy value through the different approaches proposed in this dissertation constitutes the next major stage. The first approach (described in Fig. 4b as program v.0) is based on the typical assumption found in the literature that the value of the strain energy which will accumulate in the particular elements is directly used in further proceedings. Since the strain energy value is a measure of the construction's strain in the particular elements it is used to update the Young modulus in a given element. As regards its effectiveness, this approach is compared below with the other two approaches.

Program v.1 takes into account the history of the optimization process. This means that in this approach the strain energy used in further calculations is determined as the sum of the strain energy from step 1 to the current step (bearing the $j$-th number) inclusive ( $s$ takes on values from 1 to $j$ ). This approach ensures that the optimization process is stable and the influence of the steps relatively distant from the considered step on the amount of energy accumulated in a given element diminishes.

A somewhat different way of adding up the strain energy distribution is used in program v.2. Strain energy is determined here as the arithmetic mean of the sum of the strain energy in step $j$ and in step $j-1$.
In the literature predominates the numerical approach referred to here as program v.0. All the approaches (including program v.0) presented here are based on the author's own algorithms. This means that they provide an alternative to the algorithms found in the literature. The strain energy was normalized in accordance with Eq. (37), then using Eq. (15) and Eq. (38) was written and finally, the material density was normalized. In a symbolic way the operations are shown as $\delta_{j}^{i} \Rightarrow \Pi_{j}^{i}$ in the block diagram (Fig. 4b). It should be noted that by discretely determining and normalizing the distribution of strain energy in the design domain one obtains the normalized density distribution in this domain. In the next step this distribution will be the basis for updating Young's modulus in each of the individual elements.

Since the maintenance of the mass constancy condition is the limiting condition imposed on the optimization process this condition must be and is satisfied in each of the programs. If, in addition, the penalization procedure $(P P)$ is used in any of the programs, this will be indicated each time. When $P P$ is taken into account in the computing algorithm, the obtained density distribution is again subjected to (PSSM) in order to ensure that the mass constancy condition (the right side of the block diagram in Fig. 4b) is satisfied.

The final stage in the algorithm consists in checking whether the solution obtained in the considered step $j$ is already the optimal solution (characterized by the minimal value of strain energy). If the answer is YES, the program ends. If the answer is $\mathrm{NO}$, the computing algorithm moves to the next step $(j+1)$. In order to check whether the given solution is really characterized by the minimal strain energy value, the iterative process is continued and if after a few successive steps the strain energy value increases, the optimization process is stopped and the solution for which the strain energy value is minimal is adopted as the optimal solution. Typically, when the optimization process is continued beyond the minimal strain energy solution, the mass in the given step decreases (despite the operation of (PSSM)). As a result of the mass decrement some of the bars become broken, which leads to an increase in strain energy.

What is more important, solutions with decreasing total mass cannot be taken into account in the analysis since they do not satisfy the condition of mass constancy during the optimization process. It should be added, however, that some such solutions are taken into account, but only in cases when the mass constancy condition is not satisfied to 
Table 1 Schematic diagrams of PSSM (a), PP (b) and DP (c) procedures

(a)

\begin{tabular}{lcc}
\hline $\begin{array}{l}\text { Mass constancy } \\
\text { satisfaction } \\
\text { procedure (PSSM) }\end{array}$ & $\begin{array}{c}\text { Penalization } \\
\text { procedure (PP) }\end{array}$ & $\begin{array}{c}\text { Distribution } \\
\text { procedure (DP) }\end{array}$ \\
\hline $\begin{array}{l}\text { 1) checks mass } \\
\text { constancy } \\
\text { condition, }\end{array}$ & $\begin{array}{c}\text { 1) defines negligible } \\
\text { material strain values } \\
\text { (threshold function - TF) }\end{array}$ & $\begin{array}{c}\text { 1) adds up material } \\
\text { excess (density > 1) }\end{array}$ \\
$\begin{array}{lcc}\text { 2) scales material } \\
\text { density }\end{array}$ & $\begin{array}{l}\text { 2) removes material } \\
\text { from relatively less } \\
\text { strained areas }\end{array}$ & $\begin{array}{c}\text { 2) distributes } \\
\text { collected material } \\
\text { excess }\end{array}$ \\
$\begin{array}{l}\text { 3) determines } \\
\text { lower bound value } \\
\text { (LB function) }\end{array}$ & $\begin{array}{c}\text { 3) determines lower } \\
\text { bound value (LB } \\
\text { function) }\end{array}$ & \\
$\begin{array}{l}\text { 4) distributes } \\
\text { material through }\end{array}$ & $\begin{array}{c}\text { 4) distributes material } \\
\text { through distribution } \\
\text { distribution }\end{array}$ & \\
procedure (DP) & procedure (DP) & \\
\hline
\end{tabular}

a very small degree. This is allowed since a small amount of material can be made up through postprocessing and so ultimately the mass constancy condition is satisfied. Solutions which do not satisfy this condition may be taken into account in various analyses, but only as separate solutions to serve as references for comparison purposes, e.g. in analyses aimed at determining at what amount of mass the solution is optimal.

Table 1 schematically illustrates the operation of the three procedures mentioned in Fig. 4.

\section{Mass constancy satisfaction procedure}

Table 1a schematically shows the computing algorithm which in the topology optimization procedure is responsible for keeping the mass constancy condition satisfied. The procedure is called a mass constancy satisfaction procedure (PSSM). Its particular stages are described below:

1. The (PSSM) computing algorithm has been so programmed that immediately after the density value is normalized the density of all the elements in the design domain in a given step $\mathrm{j}$ is added up. This is done in order to check whether the mass constancy condition (3.8) is satisfied. Almost always after normalization the value of available mass $m_{0}$ is not as it should be, i.e. usually $m_{j}<m_{0}$.

2. In order for condition (3.8) after normalization to be satisfied all the mass density values in the particular elements of the design domain should be rescaled. The scaling consists in multiplying each numerical value in each element of the design domain by the number calculated from the relation $\frac{\alpha \cdot N_{x} \cdot N_{y}}{\operatorname{sum}(i)}$, where $\alpha$ is a mass reduction coefficient, $N_{x}$ and $N_{y}$ stand for the number of elements along respectively orthogonal direction $x$ and $y$, and $\operatorname{sum}(i)$ is the sum of the numerical mass density values from all the finite elements in the analyzed design domain in the given step $j$ prior to scaling. As a result of scaling, mass density values in some of the elements will be higher than unity. This mass (with density higher than unity) is added up and then distributed (pt. 4) using the distribution procedure $(D P)$.

3. According to Eq. (18), densities in the design domain may assume values between lower bound $\rho_{d}$ and density $\rho$ (understood to be a normalized value). At this stage, the lower bound function $(L B F)$, which specifies limit density value $\rho_{d L B F}$, is introduced. The value is between $\rho_{d}$ and one.

4. The distribution procedure $(D P)$ uses $\rho_{d F D O}$ to distribute the material collected from the elements with density higher than unity among the elements whose density is in the range of $\left(\rho_{d F D O}, 1\right)$.

\section{Penalization procedure}

Now the part of the computing algorithm (Table 1(b)) designed to increase the effectiveness of the topology optimization process by relocating mass from less strained areas to more strained ones will be described. The program for topology optimization uses a penalization procedure $(P P)$ applied immediately after the mass constancy satisfaction procedure (PSSM). Table $1 \mathrm{~b}$ shows the main parts of $P P$ which are described in more detail below.

1. As part of the penalization procedure, negligibly small material strain values are defined through the value of the threshold function $(T F)$. The formula for the threshold function in a general way can be expressed by Eq. (19). The threshold function depends on the number $(j)$ of the iteration process step whereby its value increases in the successive steps of the optimization process. By increasing the value of $T F$ one can remove material with ever higher density, which considerably speeds up the translocation of mass in the design area and is a more effective way of moving mass than using the same constant $T F$ throughout the whole topology optimization process.

2. Since $T F$ defines the value of negligibly small material strain, the material whose density is lower than the value indicated by $T F$ can be removed from the construction. The material removed by $T F$ is added up.

3. Since the problem needs to be well conditioned it is necessary to leave such amount of material in the elements from which material has been removed which 
will ensure good problem conditioning. In this paper it is assumed that density $\rho_{d}=1 \cdot 10^{-4}$ ensures good problem conditioning. This low density value means that physically there is no material in the given element.

4. Similarly as in (PSSM) (pt. 3), the value of lower bound $\rho_{d F P}$ is defined. Constant values of $\rho_{d F P}$ are used in this paper.

5. The material totalled up earlier is then distributed using a simplified distribution procedure $(D P)$ (except for point 1 of the procedure).

If in the course of mass translocation density in some elements assumes a value higher than unity, one should iteratively apply the distribution procedure $(D P)$ in this place of the algorithm.

\section{Distribution procedure}

This procedure translocates material from elements in which density is higher than unity. It is shown schematically in Table 1(c).

1. Since density values cannot be higher than one, in the elements where this value is exceeded the value higher than one is replaced by one and the differences between one and densities higher than one are totalled up.

2. Then this total is distributed among elements whose density is in the interval of $\left(\rho_{d F D O}, 1\right)$ if the distribution procedure applies to (PSSM) and in the interval of $\left(\rho_{d F D O}, 1\right)$ if it applies to PP. The same amount of mass is added to each element. Mass is translocated within the design domain until in none of the elements within the design domain material density exceeds the value of 1 , i.e. all the density values are in the interval of $(0,1]$.

In the case when the distribution procedure is used as part of $P P$, only point 2 above is carried out.

\subsection{Steering parameters analysis and comparison of results with literature cases}

The computing algorithms for topology optimization, presented were programmed in Matlab. $20 \times 20$ elements mesh for cantilever beam is mainly used. When it is another it is described.

As an example of steering parameter analysis the following scheme was considered: the cantilever (Fig. 5) fixed at one end and loaded with a concentrated force in the middle of its height.

The lower bound $\rho_{d L B F}$ value was analyzed in this example. For v.1. program the threshold function had the form: $F P=j \cdot 0.02$ ( $j$ is the step number).
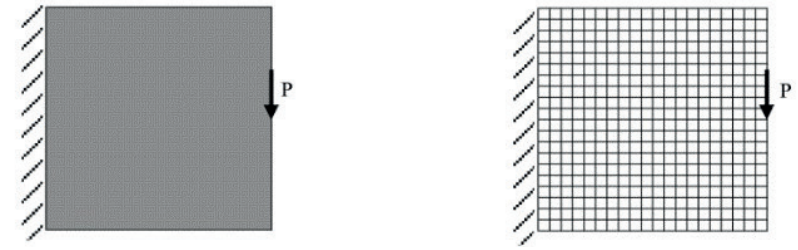

Fig. 5 Design domain: cantilever beam plus exemplary FE mesh
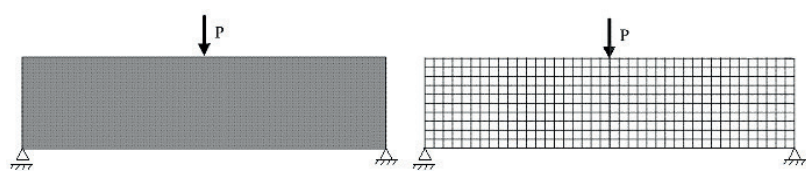

Fig. 6 Design domain: freely supported beam plus exemplary FE meshes

Solutions in which the parameter was a constant value of lower bound $\rho_{d L B F}$ and $a=0.3$ were adopted are presented below (Table 2). The solutions are characterized by the minimal topology energy value in the given process. An analysis of the solution shows that if too high values of $\rho_{d L B F}\left(\rho_{d L B F}>0.4\right)$ are used, one cannot obtain a material/ void $(0 / 1)$ distribution. It should be added that the effectiveness of the computing algorithm used to obtain $0 / 1$ topologies is better at functionally variable lower bound $\rho_{d L B F}$.

Here selected topologies obtained using program v.1 with the penalization procedure are presented and compared with literature cases.

The analyses presented below were carried out for a cantilever and a freely supported beam (Fig. 5 and Fig. 6), whereby the obtained solutions could be compared with cases reported in the literature since topology optimization problems there mainly relate to such structures.

The static diagrams of freely supported beam loaded with a concentrated force in the middle of their span is shown in Fig. 6. For the cantilever (Fig. 5) the proportion of the sides is $1: 1$ and their dimensions are $20 \times 20 \mathrm{~m}$ $(20 \times 20$ elements). For the beam the proportion of the sides is 1:6 for a division into $12 \times 72$ elements.

When comparing solutions (optimal topologies) one should bear in mind that they could be created using different numerical algorithms. Sometimes it is virtually impossible to compare two topologies since the solutions reported in the literature have no identification parameters specified. Quite often the input data used in the topology optimization computations are not explicitly given and merely selected final topologies are presented. One can only compare topologies, i.e. the shape and distribution of matter in a given structure. Sometimes, though rarely, the number of optimization steps at which the optimal solution was obtained is given. This can provide the basis for evaluating the effectiveness of the algorithms involved. 
Table 2 Topologies obtained using program v.1 with $P P$

No. $20 \times 20$ mesh topology (topology energy - step no.)

A

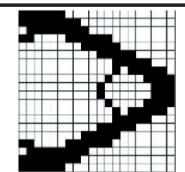

$(0.668-20)$

B

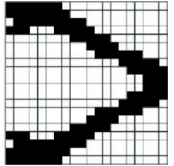

$(0.684-19)$

C

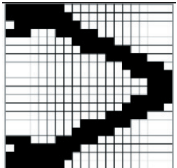

$(0.675-20)$

D

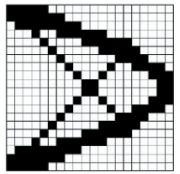

$(0.615-25)$

E

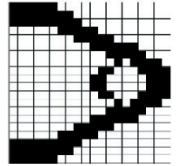

$(0.668-20)$

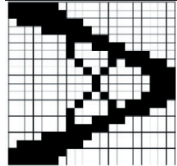

$(0.646-21)$

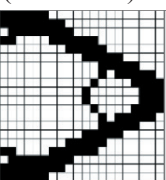

$(0.665-26)$

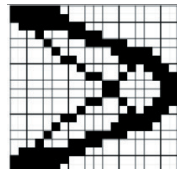

$(0.658-23)$

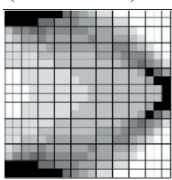

$(0.492-3)$

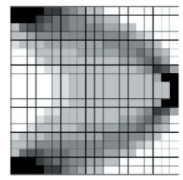

$(0.503-7)$
$40 \times 40$ mesh topology (topology energy - step no.) $\quad \rho_{d L B F}$

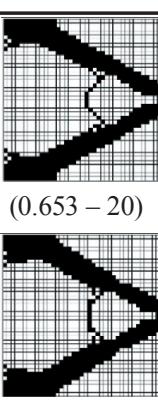

$(0.649-19)$

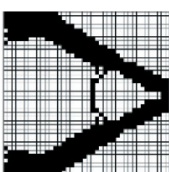

$(0.647-19)$

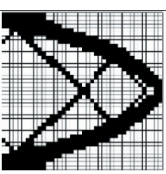

$(0.642-23)$

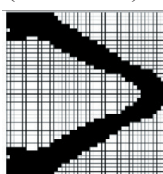

$(0.653-21)$

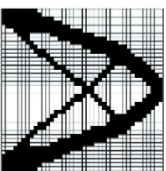

$(0.645-26)$

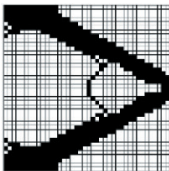

$(0.691-28)$

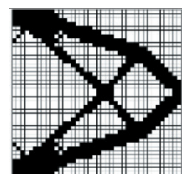

$(0.652-19)$

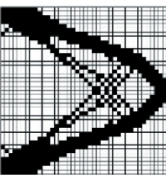

$(0.678-23)$

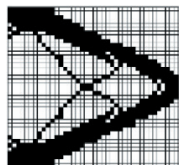

$(0.693-18)$

0.00001

0.0001

0.001

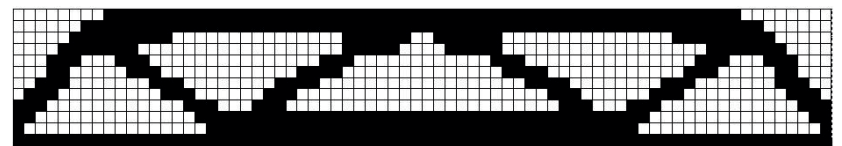

Fig. 7 Topology for scheme 1 obtained using program v.1

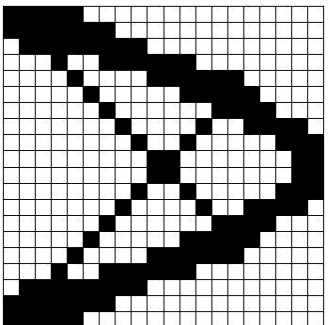

Fig. 8 Topology obtained using program v.1, shown in table 2 row D
The exemplary solution for the beam (shown in Fig. 7) is compared with the results reported in paper [8] (Fig. 1 in this paper) and in book [4] (Fig. 1.24e).

It is apparent that as regards the number and distribution of the particular bars the topology shown in Fig. 7 is similar to the solutions shown in Fig. 1 in paper [8] or Fig. 1.24e in book [4]. There is similarity in the bottom chord thickness, i.e. in the middle part between the two bars it is much thicker than in the outermost sections. Also the location of nodes in which the particular bars are joined, in both the top and bottom chords. is almost the same.

In the examples found in the literature, neither the strain energy value nor the iteration step in which the topologies were obtained is given. This makes the analysis difficult. One can only surmise that if the shapes are similar, then the strain energy values should also be comparable.

Another compared topology is the one obtained for cantilever (Fig. 8) compared with [12] (Fig. 15 - left figure). In the two cases, material in the design domain is distributed in similar areas. One can see that the middle part node with intersecting bars is located in the same place. Although the course of the longer members running left from the node towards the top chord and towards the bottom chord differs between the two topologies, the difference is small and occurs only in the places in which the members join the top chord and the bottom chord, i.e. in Fig. 8 one can see that they join the chords closer to the cantilever mounting points, whereas in Fig. 15 [12] the joints are somewhat distant from the mounting points. Very similar solution to shown in Fig. 8 one can find in [13] (Fig. 17b), where an energy-based design is used.

Below, appropriate comparisons are made for the cantilever with a sides ratio of 1:2. In the analyzed cases, at mass reduction coefficient $\alpha=0.5$ (Fig. 9) one can perceive 
some similarities, i.e. the distribution of mass in the design domain, obtained by means of the author's program (Fig. 9), is similar to the solution presented in book [14] (Fig. 4.2 in this book - a sides ratio of 1:1.5) or to the solutions found in [10] (Fig. 6b) and in [15] (Fig. 21), and in [4] (Fig. 1.23). The most strained places in the compared distributions are situated in the same areas in the design domain. Obviously, one can see a slight difference in the thickness of the particular members in the drawings, but the orientation of their axes is the same. At the constant amount of the available material to be distributed in the design domain one of the bars becomes thicker (more material is used) at the expense of the other. This can be seen in the solution shown in Fig. 9 where the bars adjoining the mounting points are thicker than the other bars (mainly the bars originating from the force application place). The amount of material in the node in which the force is applied $([4,10])$ is significantly larger than in the case of the solution shown in Fig. 9 and in [15]. Nevertheless, the arrangement of the particular structural members in the considered cases (Fig. 9) is similar.

Fig. 10 shows material distributions based on the same static scheme as the solution shown in Fig. 9, but with less available material used. In this case (Fig. 10), $\alpha=0.3$ was assumed.

The shape of the obtained solution (Fig. 10) resembles that of the topology presented in [10] (Fig. 6a in this paper). In the material distribution (Fig. 10) the location of the node constituting the center of the diagonal brace is slightly shifted towards the applied load in comparison

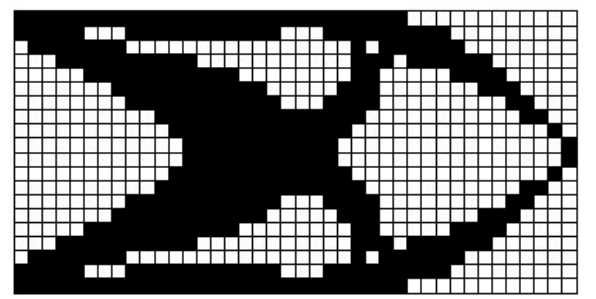

Fig. 9 Topology with sides ratio of 1:2, obtained using program v.1 $(\alpha=0.5)$

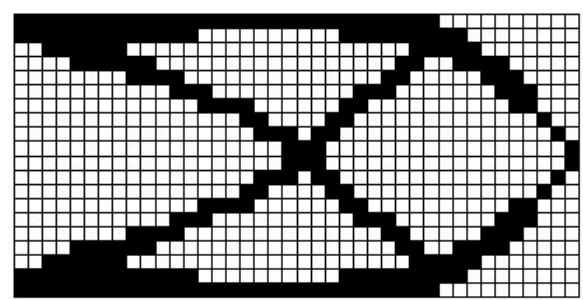

Fig. 10 Topology with sides ratio of 1:2, obtained using program v.1 $(\alpha=0.3)$ with the topology shown in [10] because the bars originating from the top and bottom chords come together in the force application point at a larger angle due to the shift of the central node of the cross. Moreover, the shift of the central node has also resulted in a change in the location of the nodes at the support, where the cross brace bars join the top and bottom chords. One should note that the distances between the particular nodes were matched in the optimization process by the algorithm to ensure comparable lengths of the particular nodes. Too long (and so thin) bars would form too fragile structural members with inadequate stiffness whereby they would not be able to carry the expected load.

Fig. 11 shows static schemes for the cantilever and the freely supported beam, but with a somewhat different loading configuration. Selected solutions (Figs. 12a-c) obtained by the author are compared with the solutions found in the literature [4], [16] and [17].

The material distribution in the topology (Fig. 12a) is almost identical with the literature one (Fig. 5.2 in [4]). The node in which the angle strut joins the top chord is located in the same area in the two cases. Some of the members, e.g. the horizontal bar in the construction's lower part (Fig. 12a), are thinner than the ones in the solution shown in Fig. 5.2 in [4], but this may be due to the different


(a)

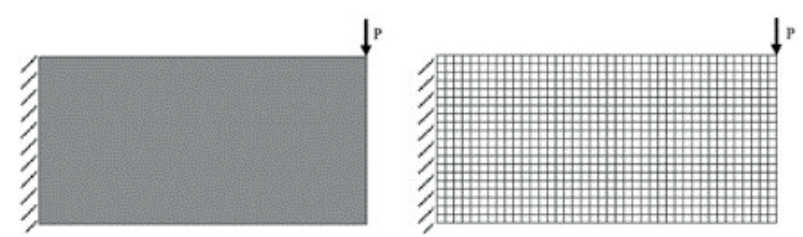

(b)

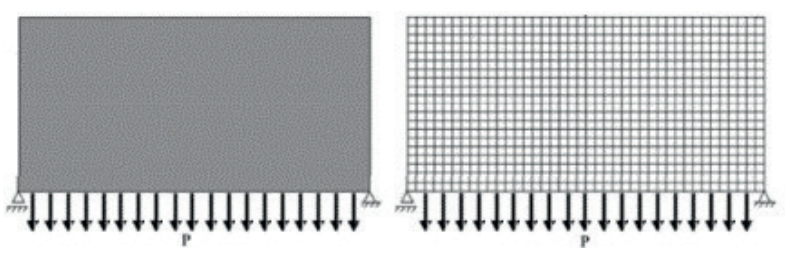

c)

Fig. 11 Design domain of cantilevers with sides ratio of 1:1.5 (a) and 1:2 (b) and of freely supported beam with sides ratio of 1:2 (c), with exemplary FE meshes 


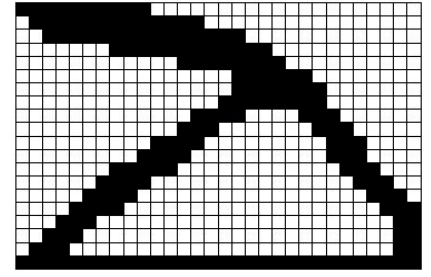

(a)



(b)

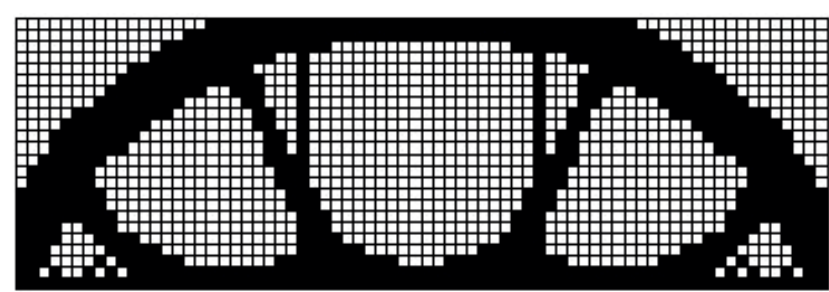

(c)

Fig. 12 Cantilever topologies obtained using program v.1 (a), (b), as well as freely supported beam topology (c)

amounts of available material used. It is difficult to determine whether the amounts were the same since in the literature case the amount is not specified. Nevertheless, the distribution of the particular members is almost identical.

Good agreement was also found for the solutions shown in Fig. 12b and Fig. 17 in [16]. In this case, the nodes in which the particular structural members come together are located in the same points of the design area. In the solution yielded by the author's program (Fig. 12b) there is relatively less material in the node in the design domain's lower part, where three bars come together, in comparison with the solution shown in Fig. 17 ([16]). Moreover, one can see that one of the bars close to this node becomes narrower as opposed to the solution found in the literature, where the thickness of this member along its length does not change. When one examines the node in which the concentrated force is applied one can see that a small diagonal has formed in the solution shown in Fig. 12b. Consequently, there is no need to use so much material in this node as in the case of the solution shown in Fig. 17 [16].

When one examines the solutions for the freely supported beam (Fig. 12c and Fig. 9b in [17]) one can notice that there is good agreement between the obtained distributions. In their shape both solutions resemble arch bridges on which traffic runs on the lower deck suspended on bars from the upper structural members. The literature solution (Fig. 9b in [17]) was obtained using a denser finite element mesh whereby more thinner bars formed in the topology. The solution obtained from the Matlab program (Fig. 12c) has fewer bars functioning as hangers on which the lower deck plate is suspended from upper chord of the structure. One can see that the slanting bars in the middle part of the design domain have been strengthened with thin vertical members. Whereas the three outermost slanting bars on the left and right side of the construction have been reduced to one thicker bar strengthened with a diagonal.

Similar to Figs. 9 and 10 solutions using Pareto method are shown in [18] (Fig. 7), these solutions are changing depends on the volume fraction.

To sum up, one can say that the optimal solution obtained by means of the program written in Matlab are very similar in their topology to the solutions reported in the literature.

\section{Application of algorithm to pavement structure - analysis of material distribution in pavement structure with hole}

As a practical example, the application of topology optimization to a selected problem from the field of road surface structure is presented. Some details concerning using topology optimization in pavement structure analysis one can find in [19] where the representative stiffness of the road pavement structure was proposed as a quantity which characterized the road pavement structure. It may be useful in designing process of the road pavement structure, because the structure may be designed properly, according to the material effort. As a representative roadway structure, pavement structure of type A on subgrade G1 with an elasticity modulus no lower than $100 \mathrm{MPa}$ at traffic class KR3 [19] is analyzed here in this paper.

The material distributions in below examples were obtained using program v. 1 with $P P . T F=j \cdot 0.02$ was used in the provided examples and in PSSM procedure the following function $\rho_{d L B F}$ was used: for the first optimization steps until $j=34 \cdot \rho_{d L B F}=j \cdot 0.02$. When $34<j<58 \cdot \rho_{d L B F}$ $=0.07+\mathrm{j} \cdot 0.0014$ and for $j>57 \cdot \rho_{d L B F}=0.07+j \cdot 0.001$.

Below, cases with a hole in the bottom course of the main crushed stone base are analyzed. It was determined how the hole can appear as a result of the undermining and washing out of the crushed aggregate. This analysis can 
be also useful in situations when it is necessary to do pipe jacking under traffic. The obtained topologies show the optimal distribution in such cases and each time one can determine whether the pipe jacking is safe or not.

Fig. 13 shows 0/1 solutions for two schemes: with and without the hole (FEM mesh is shown below as a grid.) The first of the schemes (Fig. 13a) represents a material/ void solution without the hole at mass reduction coefficient $\alpha$ equal to 0.5. Next figures (Figs. 13b-6d) present the solutions with whole (yellow color). Colors in the left side of Fig. 13a mean various layers of the pavement structure.

When one examines the structures with the hole at an increasingly smaller mass reduction coefficient, it becomes apparent that although the particular structural members are thinner, their location in the design domain does not change. The distributions are similar.

In order to precisely determine the material distributions the normalized base energy distribution, understood as the distribution of material base density, was examined. Topologies for the successive phases of the vehicle wheel movement over the hole are presented in the successive rows of Table 3 . The first column shows material/void topologies while the next columns show base density distributions in appropriate scales. It becomes apparent how the pavement structure's strain changes in the particular elements as the force moves over the hole. Using various scales (e.g. interval $0-0.2$ ) one can distinguish in detail the degree of material strain in an interval of $0-0.2$. In this case, densities higher than 0.2 are assigned black and interval $0-0.2$ is divided into 10 ranges of shades of greys.

An analysis of the solution in row A showed that material with a relatively higher density should be located under the force and in the direct vicinity of the hole. The longer the distance left and right from the most strained areas, the lower the material density. This variation is hardly



(a)

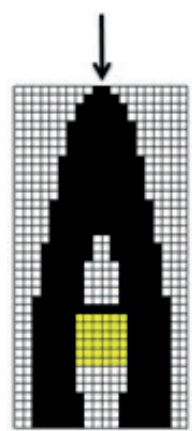

(b)

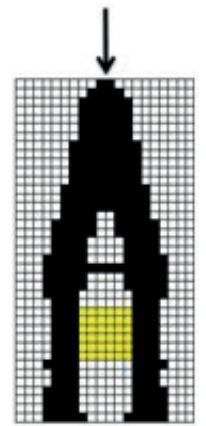

(c)

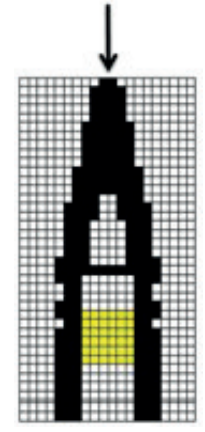

(d)
Fig. $130 / 1$ solutions at mass reduction coefficients: a) $\alpha=0.5$; b) $\alpha=0.5$; c) $\alpha=0.4$; d) $\alpha=0.3$ perceivable in the case of the $0 / 1$ distribution (row A column 2). When the material/void topology (row A column 2 ) is compared with the solution with shades of grey in the scale of $0-0.05$, close similarity becomes apparent. In the scale of $0-0.05$ one can see that the material has been distributed among the same elements as in the $0 / 1$ solution (the darkest shades of grey) and also in their surroundings (the relatively lighter shades of grey). Besides, in the scale of $0-0.02$ or $0-0.01$ one can see places above and below the hole, in which material with a much lower density than that of the other material in the vicinity of the hole is located.

The shades-of-grey distribution is a solution which shows the actual level of strain in the structure. This can be used in detail analyses of the next layers near the hole and to determine the distribution of material density in them.

The next drawings in rows $\mathrm{B}, \mathrm{C}$ and $\mathrm{D}$ show topologies for different configurations of the load above the hole. As a result of the shift of the concentrated force the different topologies were obtained.

The $0 / 1$ solution shown in row B is similar to the earlier solution in which the concentrated force was applied centrally over the hole (row A), but one can clearly see the already strengthened left part and the slightly weakened right part. This proves that the force when it shifts leftwards loads the pavement's part situated left to the hole.

As the concentrated force moved further left from hole, a distribution (row $\mathrm{C}$ ) in which most of the available material is located under the concentrated force was obtained. Only a thin structural member in the form of a support which surrounds only the hole appeared on the right side.

In row D there is a solution in which the concentrated force is shifted leftwards from the hole. As a result, a material/void topology (row D column 2) without the hole was obtained. The material distribution in row D column 2 is similar to the solution in which no hole was modelled (Fig. 6a). It is apparent that when the force is away from the hole, the presence of the hole has no effect on the material distribution. In this way one can determine the extent of the influence of the load on the particular levels of the pavement structure.

To sum up, if the force is situated centrally or almost centrally above the hole, the required material distribution is like that shown in Table 3 rows A and B. The fact that the material surrounds the hole indicates that the presence of a hole of this size in such a place is not dangerous. As the load shifts, it is transferred increasingly more vertically onto the deeper situated courses. 
Table 3 Density distribution topologies at $\alpha=0.3$ for different scale ranges

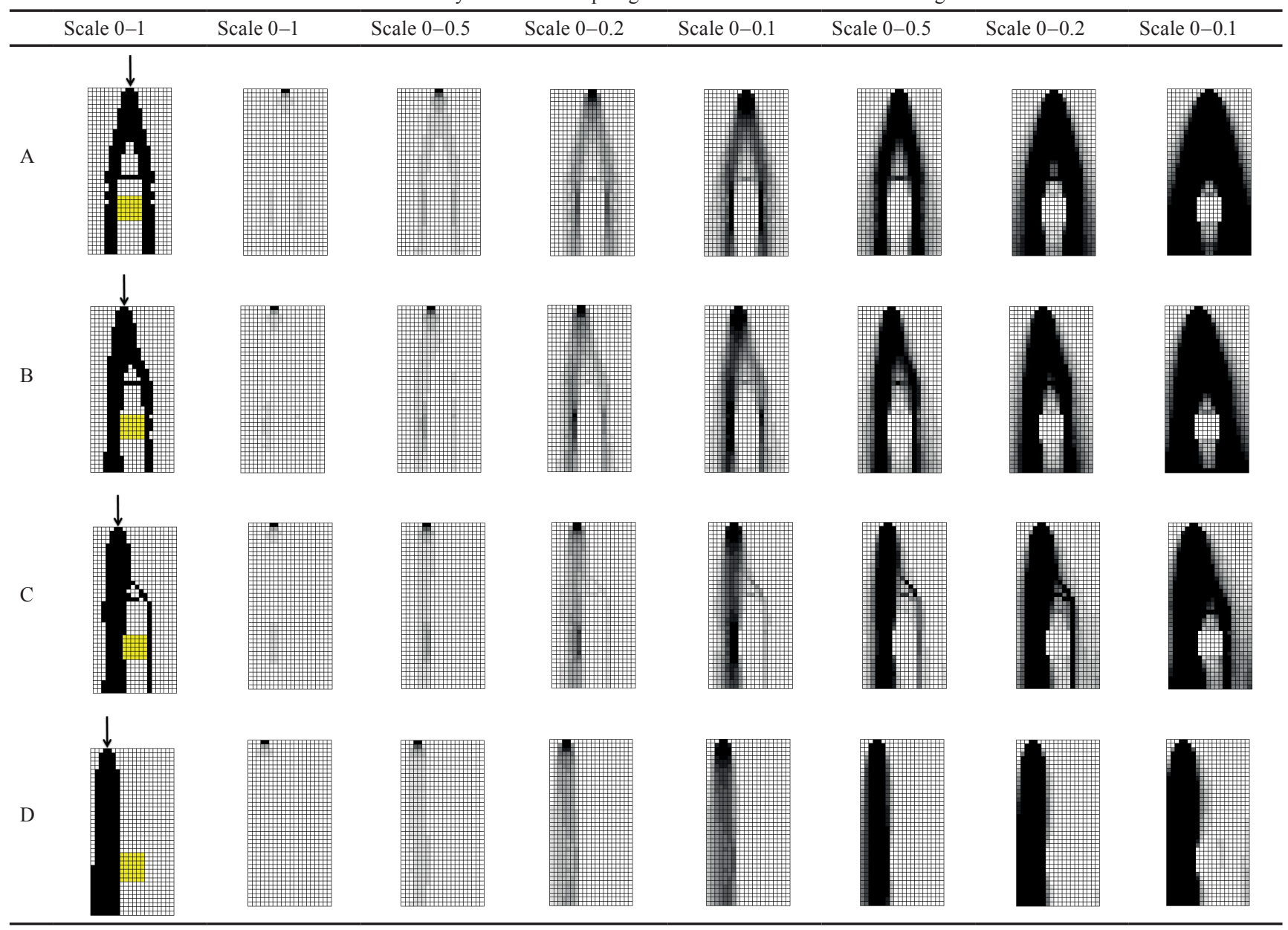

\section{Application of algorithm to analysis of tall buildings} In this section topology optimization is applied to the design of tulip-shaped tall buildings. Such building designs were proposed in the 1970s by the Polish architects W. Zalewski and W. Zabłocki [20-22]. The building shape concept derived from the optimal geometry of Michell's trusses.

As an example, an optimal topology was determined for the design domain shown in Fig. 14. The load had the form of a concentrated force which in a simplified way models the wind load dominant in the case of tall buildings.

Michell's trusses are relatively more resistant to deformation than other frame constructions subjected to the same load. This property is especially desirable in the design of tall buildings. Recently several studies devoted to the design of tall buildings have appeared. An example here is [23, 24] where a tower building model and exemplary 3D solutions obtained using a software based on systems of structural frames described by Michell were presented.

Solutions for design domains with height to length ratios of 1:2 and 1:3 are presented. Program v.1 with PP and program v.2 with $P P$ were used for the computations.

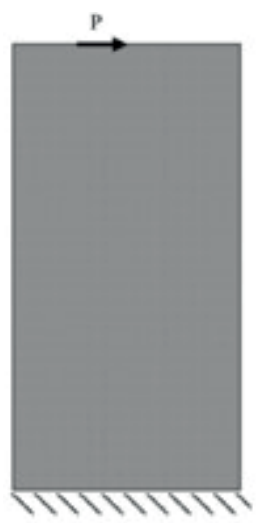

Fig. 14 Static scheme with load, used in building modelling

The solutions presented in table 4 were obtained using program v.1 with PP, in which $F P=n r^{*} 0.000125$ and $F P=$ $n r^{*} 0.00125$ were taken into account in steps $1-14$ and up to step 15 , respectively. In this way the threshold function with a discretely variable form was employed. The topologies in columns A and B in table 4 were obtained at mass coefficient $\alpha$ equal to 0.35 . The FEM mesh is shown in table 4 and 5 as a grid. Table 5 shows topologies determined using 
Table 4 Topologies obtained at sides ratio of 1:3

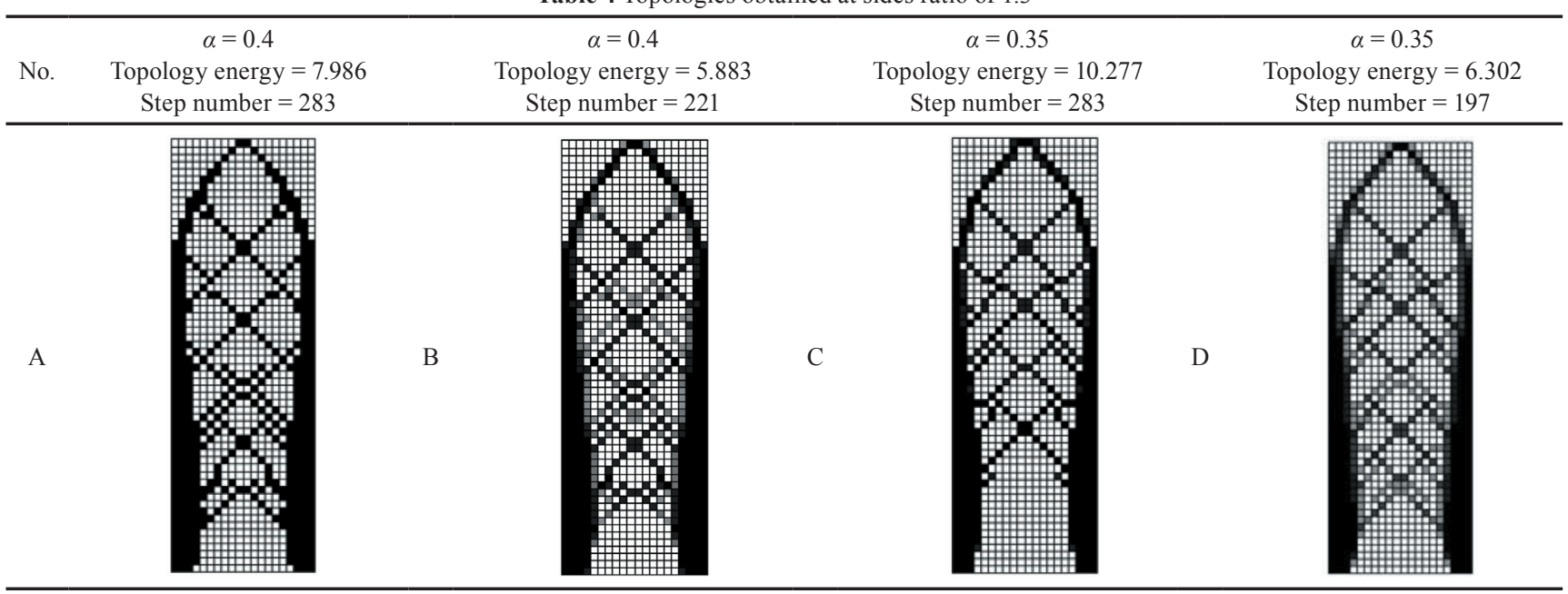

Table 5 Topologies obtained at sides ratio of 1:3

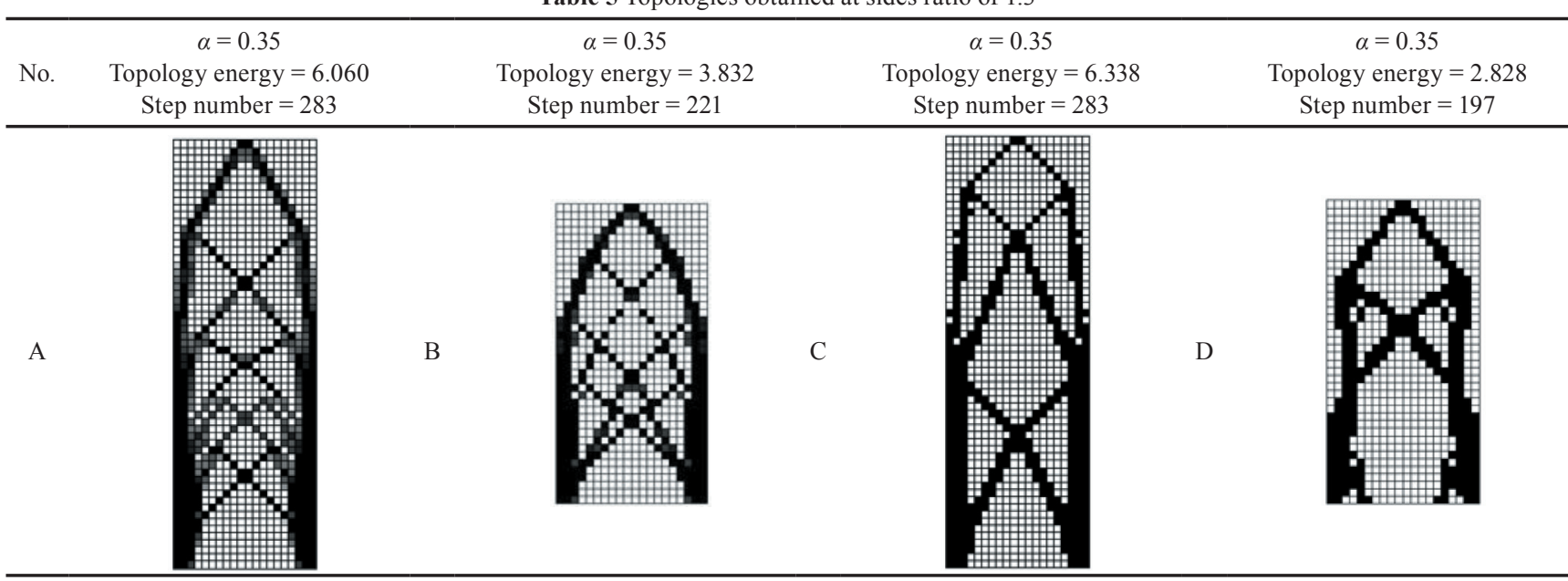

program v. 2 with $P P$ at mass coefficient $\alpha$ equal to 0.35 . In columns $\mathrm{A}$ and $\mathrm{B}$ of Table 5 there are solutions obtained for the same $T F$ as in Table 4 while in columns C and D (Table 5) there are solutions obtained using $F P=n r^{*} 0.02$.

The topology shown in Table 4A is a material/void solution. It includes numerous bars connecting the exterior walls on the left and right, which are so situated that the structure carries the load in an optimal way. The obtained solutions (Tables 4 and 5) allude to Michell's trusses.

At a slightly lower value of the mass reduction coefficient, i.e. $\alpha=0.35$, a material/void solution (Table 4C) was obtained, but with a smaller number of bars strutting the exterior walls (especially in the lower part of the structure) than in the topology shown in Table 4A, which is due to the smaller amount of available mass. This demonstrates that in order to acquire proper information for design purposes one should study a proper number of cases for different available mass and carry out an analysis of the objective function value.
In this research it was shown earlier that optimal topologies characterized by the lowest strain energy do not always have $0 / 1$ distributions. Also in this section, besides solutions of the material/void type (Table 4A and C), optimal topologies with varying material density distribution (Table 4B and D), characterized by minimal strain energy in the considered processes, are presented. Their material/ void distributions are shown in Table 4A and C.

A comparison of the solutions (Table $4 \mathrm{~A}$ versus $\mathrm{B}$ and $\mathrm{C}$ versus D) shows differences in the number of bars which formed in the central part of the design domain between the two exterior walls. The topologies shown in columns B and D contain more elements built from rarefied material with a density of $0-1$. It appears from the solutions presented in table 4 that the thickness of the exterior walls changes along their height: the walls become thinner with height.

Table 5 shows optimal solutions with the lowest strain energy for the given optimization process. In columns A and $\mathrm{C}$ a width/height ratio of 1:3 was adopted while in 
columns $\mathrm{B}$ and $\mathrm{D}$ this ratio is $1: 2$. An analysis of the optimal solutions included in Table 5, similarly as those in Table 4) shows that the structures should be made from bars differing in their material parameters and thickness. In some members of the above solutions one can discern a core (the black color) surrounded with material characterized by relatively worse parameters (the shades of grey).

The topologies presented in Table 5 columns C and D, which were obtained using a slightly different TF, constitute an interesting group of solutions. At different sides ratios one can see some similarity to Michell's trusses, i.e. in the narrowing of the structure in its upper part. The solutions have a conical shape, i.e. they are wider at the base and taper in their upper part. In the topology shown in Table 5 column D, members in the form of buttresses formed in the structure's mounting part. They resemble the buttresses supporting the bearing walls in high masonry structures. In the analyzed solutions one can also notice the changing thickness of the (exterior) bearing walls. The solutions contained in Table 5 columns $\mathrm{C}$ and $\mathrm{D}$ do not have a large number of bars in the central part of the structure as it is the case in the topologies presented in columns A and B of Table 5.

\section{Balcony cantilevers}

The cantilever is a bearing element on which the balcony slab rests. Usually these balconies slab rests on stone or cast iron cantilevers secured in the wall. Such balconies used to be frequently made in the second half of the 19th century and at the beginning of the 20th century. They were characterized by good aesthetics.

For optimal balcony cantilever topology computations it was assumed that the load from the balcony slab was continuously transferred to the cantilever (Figs. 15a and 16a). Two schemes differing in their ratio of the sides (in Fig. 15 the proportion between the design domain's height and width is $1: 1$ whereas in Fig. 16 it is 1:2) were analyzed. The obtained topologies show the optimal shape for the analyzed static diagrams using program v1 with $P P$.

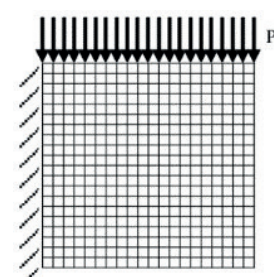

(a)

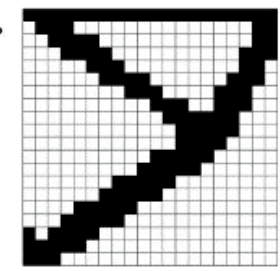

(b)

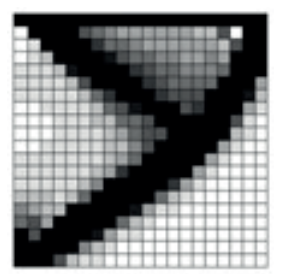

(c)
Fig. 15 Static diagram (a), 0/1 solution obtained in step 91 (b) and solution in scale of $0-0.03$ obtained in step 91 (c)

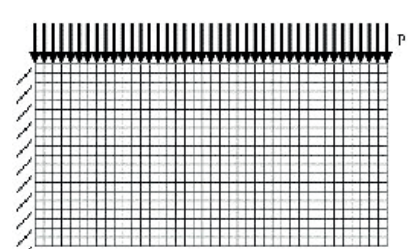

(a)

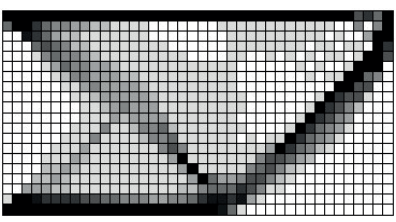

scale $0-0.14$

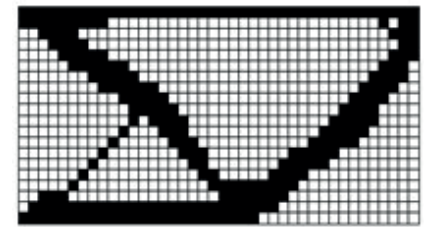

(b)

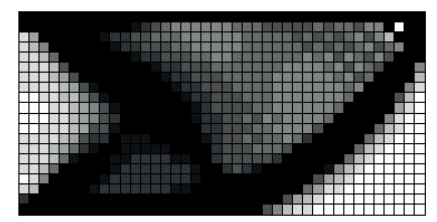

(c)

scale $0-0.03$

Fig. 16 Static diagram (a) 0/1 solution obtained in step 94 (b) and solution obtained in step 94, shown in two scales (c)

In Figs. $15 \mathrm{~b}$ and $16 \mathrm{~b}$ there are solutions of the material/ void type. In addition, Fig. 15c shows the obtained solution in a scale of $0-0.03$. As a result, the microstructure of the construction becomes visible. Today it is possible to exploit such a microstructural construction, as demonstrated in, e.g., [25] where a porous cement matrix with inclusions was analyzed and it was found that its properties depend mainly on density. Thus a topology with varied density can serve as the basis for designing a structure with from point to point variable properties, which means that one can shape, e.g., a cantilever from a material the properties of which are shown in the topology drawing.

The obtained optimal cantilever structure with a $0 / 1$ distribution should consist of members in the form of bars and struts (Figs. 15b and 16b). The solutions presented in Figs. 15c and 16c show a heterogeneous distribution. Particularly in Fig. 15c one can see that at a ratio of 1:1, material with varied density fills a considerable part of the area above the slanting bar, which suggests that the cantilever should be designed as shown in Fig. 15b, or it can be a solid cantilever where there will be material also above the slanting bar. Thus the topology shown in Fig. 15c can be regarded as corresponding to the cantilever structure where balconies slab rests on stone. Fig. 16c shows, in two different scales, solutions corresponding to the cast iron structure consisting of many bars. By analyzing Fig. 16c one can form an opinion about the structure's material needs and then shape it taking into account the solutions obtained from topology optimization. One should note that the cantilevers supporting the balcony slab in old tenement houses correspond to the optimal solutions. Hence one can conclude that in the past designers would produce designs which were both optimal and aesthetic. 


\section{Conclusions}

A variational formulation of the topology optimization problem, where the compliance of the structure is expressed in the form of an objective functional, was adopted in this study. Taking into account the equivalence of compliance and strain energy, strain energy was minimized under constraints imposed on the body mass. The optimization problem solution was formulated as an analysis of sequences of topologies and corresponding objective functional values. The sequences are the solutions obtained under the adopted optimization process control parameters. A computer program, taking into account algorithm paths (Chapter 2), was created in Matlab. The required optimal distribution of

\section{References}

[1] Bendsøe, M. P. "Optimal shape design as a material distribution problem", Structural Optimization, 1, pp. 193-202, 1989. https://doi.org/10.1007/BF01650949

[2] Ramm, E., Bletzinger, K. U., Reitinger, R., Maute, K. "The challenge of structural optimization", In: Topping, B. H. V., Papadrakakis, M. (eds.) Advanced in Structural Optimization, Civil-Comp Press, Edinburgh, Scotland, 1994, pp. 27-52.

[3] Bendsøe, M. P., Sigmund, O. "Material interpolation schemes in topology optimization", Archive of Applied Mechanics, 69, pp. 635-654, 1999.

https://doi.org/10.1007/s004190050248

[4] Bendsøe, M. P., Sigmund, O. "Topology Optimization - Theory, Methods and Applications", 2nd ed., Springer, Berlin, Germany, 2003.

https://doi.org/10.1007/978-3-662-05086-6

[5] Kutyłowski, R. "On nonunique solutions in topology optimization", Structural and Multidisciplinary Optimization, 23(5), pp. 398-403, 2002.

https://doi.org/10.1007/s00158-002-0200-8

[6] Guedes, J. M., Taylor, J. E. "On the prediction of material properties and topology for optimal continuum structures", Structural Optimization, 14(2-3), pp. 193-199, 1997. https://doi.org/10.1007/BF01812523

[7] Kaveh, A., Hassani, B., Shojaee, S., Tavakkoli, S. M. "Structural topology optimization using ant colony methodology", Engineering Structures, 30(9), pp. 2559-2565, 2008. https://doi.org/10.1016/j.engstruct.2008.02.012

[8] Sigmund, O. "A 99 line topology optimization code written in Matlab", Structural and Multidisciplinary Optimization, 21(2), pp. 120-127, 2001. https://doi.org/10.1007/s001580050176

[9] Andreassen, E., Clausen, A., Schevenels, M., Lazarov, B. S., Sigmund, O. "Efficient topology optimization in Matlab using 88 lines of code", Structural and Multidisciplinary Optimization, 43(1), pp. 1-16, 2011

https://doi.org/10.1007/s00158-010-0594-7 material in the pavement structure under the wheel load for a special case was analyzed: the effect of a hole in the base course on the distribution of material in the structure, needed to carry the load was examined. The second considered example deals with the tall buildings: an analysis of obtained topologies of such buildings confirmed the usefulness of topology optimization in the preliminary design of tall building structures. The third example shows balcony cantilevers as a material-voids solutions and the structure made of microstructure material. An analysis of balcony cantilevers indicated that the balconies made in the 19th and 20th centuries often show the characteristics of optimal structures.

[10] Suresh, K. "A 199-line Matlab code for Pareto-optimal trading in topology optimization", Structural and Multidisciplinary Optimization, 42, pp. 665-679, 2010. https://doi.org/10.1007/s00158-010-0534-6

[11] Sokół, T. "A 99 line code for discretized Michell truss optimization written in Mathematica", Structural and Multidisciplinary Optimization, 43(2), 181-190, 2011. https://doi.org/10.1007/s00158-010-0557-z

[12] Bruggi, M., Venini, P. "A mixed FEM approach to stress-constrained topology optimization", International Journal for Numerical Methods in Engineering, 73(12), pp. 1693-1714, 2008.

[13] Bruggi, M. "Topology optimization with mixed finite elements on regular grids", Computer Methods in Applied Mechanics and Engineering, 305, pp. 133-153, 2016. https://doi.org/10.1016/j.cma.2016.03.010

[14] Xie, Y. M., Steven, G. P. "Evolutionary structural optimization", Springer, London, UK, 1997. https://doi.org/10.1007/978-1-4471-0985-3

[15] Harasaki, H., Arora, J. S. "A new class of evolutionary methods based on the koncept of transfer red force for structural design", Structural and Multidisciplinary Optimization, 22, pp. 35-56, 2001. https://doi.org/10.1007/s001580100123

[16] Sui, Y., Peng, X., Feng, J., Ye, H. "Independent continuous and mapping method of structural topology optimization based on global stress approach", Frontiers of Mechanical Engineering in China, 5, pp. 130-142, 2010 https://doi.org/10.1007/s11465-010-0011-3

[17] Bruns, T. E. "A reevalution of the SIMP method with filtering and an alternative formulation for solid-void topology optimization", Structural and Multidisciplinary Optimization, 30(6), pp. 428-436, 2005. https://doi.org/10.1007/s00158-005-0537-x

[18] Deng, S., Suresh, K. "Multi-constrained topology optimization via the topological sensitivity", Structural and Multidisciplinary Optimization, 51, pp. 987-1001, 2015. https://doi.org/10.1007/s00158-014-1188-6 
[19] Kutyłowski, R., Szwechłowicz, M. "Topology optimization as a tool for road pavement structure analysis", In: Proceedings of the 3rd Polish Congress of Mechanics (PCM) and 21st International Conference on Computer Methods in Mechanics (CMM), Gdansk, Poland, 2016, pp. 321-325.

[20] Lewiński, T., Sokół, T., Graczykowski, C. "Michell Structures", Springer, Cham, Switzerland, 2019. https://doi.org/10.1007/978-3-319-95180-5

[21] Zalewski, W. "Strength and lightness - The muses of a structural designer", Architektura, 74(11), pp. 94-95, 2000. (in Polish).

[22] Zalewski, W., Zabłocki, W. "Engineering inspirations in shaping tall buildings", In: Lightweight Structures in Civil Engineering, Proceedings of the International Symposium, Warsaw, Poland, 2002, pp. 109-118.
[23] Prichard, T. J., Gilbert, M., Tyas, A. "Plastic layout optimization of large-scale frameworks subject to multiple load cases, member selfweight and with joint length penalties", presented at the 6th World Congress of Structural and Multidisciplinary Optimization, Rio de Janeiro, Brazil, May, 30 - June, 3, 2005.

[24] Lewiński, T., Czarnecki, S., Dzierżanowski, G., Sokół, T. "Topology optimization in structural mechanics", Bulletin of the Polish Academy of Sciences, Technical Sciences, 61(1), pp. 23-37, 2013. https://doi.org/10.2478/bpasts-2013-0002

[25] Kligys, M, Sinica, L. M., Sezemanas, G. "The Influence of Some Surfactants on Porous Concrete Properties", Materials Science, 13(4), pp. 310-316, 2007. 